\title{
Kompakte gasisolierte Schaltanlagen und ihr Nutzen für den Anwender
}

\author{
D. Füchsle
}

Neue technische Erkenntnisse, intensive Entwicklungs- und Forschungsaktivitäten führen zu immer kompakteren gasisolierten Schaltanlagen (GIS), welche mit wenig Aufwand in immer kürzerer Zeit geliefert und montiert werden können. Unterstützt von gut funktionierenden Forschungszentren und Entwicklungsabteilungen, welche mit modernsten Hilfsmitteln ausgestattet sind, können Geräte und Systeme so dimensioniert werden, dass mit möglichst wenig Energiebedarf bei den Leistungsschaltern sowie einer effizienten Dimensionierung des Systems eine deutliche Reduktion des eingesetzten Isoliergases erzielt werden kann. Dieser Prozess wird zudem von kontinuierlich steigenden Anforderungen der Kunden an kürzere Lieferzeiten als auch von steigenden Anforderungen an die Umweltbedingungen getrieben. Schlussendlich führt dies bei höheren Spannungsebenen im Übertragungsbereich (>170 kV) dazu, dass komplette Felder als fabrikgeprüfte Einheiten transportiert werden können, welches im Bereich der Sub-Transmission ( $\leq 170 \mathrm{kV}$ ) bereits heute schon Standard ist. Die gewohnten kurzen Montageund Inbetriebsetzungszeiten lassen sich daher auch für die höheren Spannungsebenen realisieren.

Mit der neuesten Generation der ELK-14-Produktreihe, die für eine Nennspannung von 253 kV, Kurzschlussströme von 50 kA und Nennströme bis 3150 A dimensioniert ist, sind die wesentlichen Anforderungen an die geänderten Bedingungen erfüllt worden. Neueste computerunterstützte, dreidimensionale Dimensionierungswerkzeuge sowie mehr als 50-jährige GIS-Forschungs- und Entwicklungserfahrung haben zu kontinuierlichen Produktverbesserungen und -optimierungen geführt, die die neuesten Produktgenerationen nicht nur kompakter, sondern auch sicherer in ihrem Betrieb machten, da neue technische Erkenntnisse für Verbesserungen sorgten.

Ein wesentlicher Erfolgsfaktor in diesem Entwicklungsprozess ist der Einsatz eines Selbstblasschalters mit seiner deutlich reduzierten Antriebsenergie. Gepaart mit dem bewährten Federspeicherantrieb mit hydraulischer Kraftübertragung vom Typ HMB und seiner deutlich reduzierten maximal benötigten Antriebsenergie zur Lichtbogenlöschung bis zu einem Faktor 4 gegenüber einem Blaskolbenschalter mit vergleichbarer Leistung, können nicht nur die Gebäudeauslegungen aufgrund der dynamischen Kräfte optimiert werden, sondern auch der benötigte Materialeinsatz des Produktes optimiert werden. Das Weglassen eines Kammerisolators beim Leistungsschalter bewirkt eine deutliche Reduktion des Schalterdurchmessers bei gleichzeitiger Steigerung der Dauerstrombelastbarkeit aufgrund der besseren Wärmeabführung im Bereich der Nennstromkontakte.
Zudem führte ein Innovationsschritt zu einer drastischen Höhenreduktion, welche durch dezentrale Antriebe für Trenn- und Erdungsschalter erreicht wird, die über flexible Wellen miteinander verbunden sind. Dadurch können sämtliche Antriebe vor dem Feld in einem so genannten Antriebsschrank unter dem eigentlichen Feldsteuerschrank angebracht werden und zusätzliche "Catwalks" vermieden werden.

Das Resultat einer derart kompakten Bauweise für ein $245 \mathrm{kV}$ GISFeld ist die Möglichkeit einer kompletten Feldmontage innerhalb der Fabrik, inklusive der Feldsteuerung und den grundlegenden Feldverriegelungen. Der feldweise Transport hat zudem auch noch einen weiteren positiven Umweltaspekt, denn die Anzahl der Flanschverbindungen, die vor Ort noch zu überprüfen sind und damit das Risiko für Gas-Leckagen bergen, kann auf ein Minimum reduziert werden. Darüber hinaus ist die Menge an $\mathrm{SF}_{6}$-Gas in der neuesten Generation der ELK-14 aufgrund ihrer Kompaktheit - je nach Anlagenkonfiguration - bis zu $20 \%$ geringer.

Die Kompaktheit der neuesten GIS-Generationen und die damit einhergehende Größenreduktion hat zudem einen massiven Einfluss auf die Größe der Gebäude für eine gasisolierte Schaltanlage, die, je nach Konfiguration, bis zu $40 \%$ gehen kann. Dies wiederum spart Kosten bei der Gebäudeerstellung, dem Gebäudematerial und den Betriebskosten und steigert darum wiederum die Energieeffizienz des Gesamtsystems.

\section{Zusammenfassung}

Die Kompaktheit und die Verwendung der neuesten Technologien in den neuesten GIS-Generationen bieten folgende wesentliche Kundenvorteile:

- Optimale Zugänglichkeit aufgrund der Tatsache, dass alle Antriebe auf der Vorderseite eines Feldes angebracht sind.

- Verbesserte Umweltfreundlichkeit durch geringeren Einsatz von $\mathrm{SF}_{6}$, Reduktion von Flanschverbindungen und Anzahl Kuppelstellen auf der Anlage.

- Reduktion des Materialeinsatzes und der damit verbunden Umweltbelastung.

- Der feldweise Transport reduziert die Inbetriebsetzungszeiten, da sämtliche Funktionen bereits in der Fabrik des Herstellers geprüft wurden. Damit wird das Risiko einer Projektverzögerung deutlich reduziert.

- Deutliche Einsparungen in der Gebäudedimensionierung aufgrund der Kompaktheit und der Verwendung neuester SchalterTechnologien.

Füchsle, Dieter, Dipl.-Ing., ABB Schweiz AG, High Voltage Products, Brown Boveri-Strasse 5, 8050 Zürich, Schweiz (E-Mail: dieter.fuechsle@ch.abb.com) 